\section{P381 CLINICAL, EPIDEMIOLOGICAL CHARACTERISTIC OF PATIENTS WITH ACUTE RESPIRATORY TRACT INFECTIONS APPLIED TO THE MURATSAN UNIVERSITY HOSPITAL COMPLEX IN JANUARY 2019, ARMENIA}

\begin{abstract}
1,2,3Hripsime Apresyan*, 'Hasmik Bichakhchyan, 'Elmira Balasanyan, 'Luiza Hakobyan, ${ }^{1}$ Hermine Zargaryan, 'Lilit Barseghyan, ${ }^{1}$ Mark Grigoryan, ${ }^{2}$ Liana Nazaryan, ${ }^{2}$ Karmella Poghosyan, ${ }^{2}$ Bagrat Baveyan, ${ }^{1}$ Garnik Avetisyan, ${ }^{1,2}$ Vigen Asoyan. ${ }^{1}$ Yerevan State Medical University after Mkhitar Heratsi, Yerevan, Armenia; ${ }^{2}$ Muratsan University Hospital Complex, Yerevan, Armenia; ${ }^{3}$ Wigmore Clinic, Yerevan, Armenia
\end{abstract}

\subsection{6/archdischild-2019-epa.727}

Background Muratsan University Hospital Complex(UHC) is multidisciplinary hospital with 260 bed and has Pediatric, surgical, toxicological, traumatological, ICU, emergency, neonatal pathology, mandibulofacial surgical, Otolaryngologic departments.

Our goal was to describe the clinico-epidemiological characteristic of patients with acute respiratory tract infection (ARTI) applied to the Muratsan UHC in January 2019.

Acute respiratory tract infections are the most common childhood infections worldwide, with close to $100 \%$ of children being infected during the first years of life. Whereas the vast majority of viral respiratory infections are mild and selflimiting, more severe disease leads to the hospitalization.

Methods and materials We used the medical charts of patients (up to 18 year) applied to the Muratsan University Hospital Complex in January 2019.

Results In January 20193572 patients applied to the Muratsan UHC, 1117 of them were hospitalized. 1523(58.7\%) outpatients from 2594 and 603(53.9\%) inpatients from 1117 had symptoms of Acute respiratory tract infection.

Analyzing outpatients with ARTI, we mentioned, that 688 $(43.2 \%)$ were female, and $865(56.8 \%)$ - male. The 1021 $(67 \%)$ were from Yerevan and 502(33\%) from regions. Patients were divided to four age groups $0-1$ years old 388 (25.5\%), 1-3 years 565(37,1\%), 3-7 years $422(27.7 \%)$, and above 7- 148 (9.7\%). 1247(81.9\%) patients of 1523 applied up to 3 days of diseases, $181(11.9 \%)-4-5^{\text {th }}$ days and $95(6.2 \%)$ more than $6^{\text {th }}$ day. High temperature up to $38.0^{\circ} \mathrm{C}$ had 1182 patients, $38.1-40.0^{\circ} \mathrm{C}-336$ and more than $40.1-5,77.6 \%$, $22.1 \%$ and $0.3 \%$ respectively. The main symptoms at the moment of admission were cough 1025(67.3\%), catarrhal symptoms- 575(37.8\%), vomiting $-28(1.8 \%)$ and diarrhea- 12 (0.89\%).

Chest X-ray were done in 152 cases, and in 50 cases pneumonia was established (1.9\%).

Among hospitalized patients pneumonia had 191(17.1\%)

Conclusion We find out that patients with ARTI applied in first 3 days of disease, with subfebrile temperature and cough and were children up to 7 years old(90.7\%). Only $1.9 \%$ of outpatients had pneumonia.

As we mentioned children who admitted to the hospital didn't have severe complications. And education of population and some training courses for pediatricians of primary care can decrease hospital application rate.

\section{P382 THINKING OUTSIDE THE POX}

Claire Reynolds*, Molly Cremin, Susanna Felsenstein. Department of Paediatrics, Cork University Hospital, Cork, Ireland

10.1136/archdischild-2019-epa.728

\section{CASE REPORT}

Background Varicella zoster virus (VZV) causes chicken pox and shingles. Neurological manifestations occur in both illnesses. An exanthem is usually present except in the immunocompromised and elderly.

Case A 14 year old girl presented with headache, fever, vomiting and photophobia, though no meningism or altered consciousness. She was febrile, Glasgow Coma Scale was 15, she was fully orientated and conversing freely. Neurological exam revealed brisk deep tendon reflexes of all 4 limbs and ankle clonus bilaterally. The remainder of her examination was normal. There was no significant past medical history. Her vaccinations were up to date. Baseline bloods reported normal complete blood count, renal and hepatic profile, thyroid function tests and inflammatory markers. CT brain was normal. Lumbar puncture revealed a CSF protein of $1.4 \mathrm{~g} / \mathrm{L}$, glucose of $2.3 \mathrm{mmol} / \mathrm{L}, 356 \times 10^{\wedge}$ 9/L WBC, $99 \%$ of which lymphocytes. MRI brain and spine and EEG were normal. The patient underwent an extensive infectious diseases work up for viral, bacterial and fungal etiologies, autoimmune work up and flow cytometry of CSF in order to outrule a primary CNS malignancy. She received acyclovir and cefotaxime as empiric treatment for infectious meningoencephalitis. Given anamnestic potential for TB exposure, antimycobacterial combination therapy also was commenced. Subsequently, AFB stain and Mtb PCR on CSF were negative, as were bacterial and fungal PCRs and cultures. A chest XR, tuberculin skin test and IGRA all yielded a negative result. CSF virology identified varicella zoster virus positive PCR. The patient was treated for VZV meningoencephalitis most likely due to VZV reactivation following primary infection in early childhood. Flow cytometry of CSF leukocytes identified a high proportion (12\%) of double negative $\mathrm{T}$ cells, with normal flow cytometry of peripheral blood. Her recovery was complicated by bilateral anterior uveitis two months after initial presentation which resolved on topical steroids. She completed a 21 day course of Aciclovir and recovered fully.

Conclusion We present a rare case of aseptic meningitis caused by VZV reactivation without exanthem in an immunocompetent patient. This case is instructive in posing a wide differential diagnosis including the possibility of viral meningoencephalitis, an evolving autoimmune process, postviral complications or treatment related adverse effects; and illustrates the need for a better understanding of the role of certain lymphocyte subsets in infectious and autoimmune disorders involving the central nervous system.

\section{P383 STROKE IN CHILDREN; CONSIDER PARVOVIRUS B19 INFECTION}

${ }^{1}$ Kevin Conlon*, ${ }^{1}$ Molly Cremin, 'Daragh Finn, ${ }^{2}$ Clodagh Ryan, ${ }^{1}$ Michael Moore ${ }^{1}$ Niamh McSweeney. ${ }^{1}$ Cork University Hospital, Cork, Ireland; ${ }^{2}$ Mercy University Hospital, Cork, Ireland

\subsection{6/archdischild-2019-epa.729}

Introduction Common infections can transiently increase the risk of childhood arterial ischaemic stroke (AIS) by inducing an inflammatory state resulting in endothelial injury. We report a case of internal carotid artery territory ischaemic stroke in the context of Parvovirus B19 infection in a previously-well child. Normocytic, normochromic anaemia and reduced reticulocyte count were detected on investigation 
Case presentation We report a 2.5 year-old Caucasian female who presented with sudden onset right- hemiplegia and irritability. She had minor head trauma earlier that day but no loss of consciousness. She had a history of recurrent upper respiratory tract infections, fatigue, poor diet and constipation over the preceding months but no recent illnesses. These symptoms followed a trip to Rwanda where she had a bout of gastroeneteritis. Blood tests were noteworthy for a reduced Haemoglobin of $6.4 \mathrm{~g} / \mathrm{dl}$ with a repeat $\mathrm{Hb}$ of $5.7 \mathrm{~g} / \mathrm{dl}$ (normocytic, normochromic), reduced reticulocyte count of 3.9 and platelet count of 614 but normal liver function. Borellia, Varicella, Mycoplasma and Parvovirus B19 serology were negative

CT Brain was normal. A time-of-flight MR angiography of the circle of Willis showed diffuse ischaemia within the cortex of the left cerebral hemisphere involving the frontal, parietal and occipital lobes. No large vessel occlusion or haemorrhage were seen, and intracranial and extracranial carotid and vertebral arteries were normal. Echo and bubble study was normal.

Abdominal ultrasound showed a calculus in the neck of the gallbladder without cholecystitis. Repeat Parvovirus B19 IgG was positive

Discussion Previous case-control studies have suggested that the risk of childhood arterial ischaemic stroke is increased transiently in the context of acute infection. A multi-centre study in 2017 (Vascular effects of Infection in Paediatric Stroke, VIPS) Study found serological evidence of Parvovirus B19(PVB19) in $6 \%$ of cases of childhood arterial ischaemic stroke. Parvovirus B19 is a DNA virus can cause sub-clinical infection or manifest with flu-like symptoms. Infection is typically mild, but complications can include chronic anaemia.

It has been hypothesised in previous studies that Parvovirus may injure cardiac and arterial endothelium, promoting thrombus or arterial stenosis. However, it is worth noting that neither stenosis nor thrombus were detected in our patient.

Conclusion Parvovirus B19 is an important consideration in cases of stroke in children in particular in the context of retiuculocytopenia and normocytic normochromic anaemia.

\section{P384 MORE THAN MEETS THE EYE}

Joana Figueirinha* , Sara Rolim, Ana Sofia Gomes, Filipa Pinto, Paulo Teixeira. Centro Hospitalar do Médio Ave, Vila Nova de Famalicão, Portugal

\subsection{6/archdischild-2019-epa.730}

Background A teenager with periorbital edema can be a challenging diagnosis since there are numerous diseases that can have this sign, both infectious and non-infectious

Case-report A 14-year-old female presents in the Emergency Department (E.D.) with a bilateral periorbital edema and fatigue that started 5 days before. She denied itchiness, visual complaints, ocular pain or eye discharge. There was no eyelid inflammation and no peripheral edema. Blood tests were normal and proteinuria was excluded. She was sent home with an antihistamine, but came back to the E.D. 3 days later (day 8 of disease) with the same complaint. Apart from the periorbital edema and the fatigue, she had pharyngitis without tonsillar exudate, elevated aminotransferases and lactate dehydrogenase and relative lymphocytosis (52.9\%). The PaulBunnel test was negative and the tests for viral capsular antigens (VCA IgM and IgG) for Epstein-Barr virus (EBV) were negative. She developed fever between day 8 and 10. She was referred to a Pediatric consultation and reevaluated at day 11 .
She still maintained periorbital edema and had a painful palpable liver edge. The abdominal ultrasound showed hepatosplenomegaly. She repeated the blood tests two weeks after and had lymphocytosis (5190/uL; 66.5\%), elevated aminotransferases and lactate dehydrogenase, and she was EBV VCA IgM and IgG positive, which confirmed active infectious mononucleosis. The testing for autoimmune disease, thyroid disease, and human immunodeficiency virus (HIV) and hepatitis viruses were negative.

Conclusion EBV can affect virtually any organ system and periorbital edema can be the first symptom of infectious mononucleosis. This is an atypical case for there was a lack of other more classic symptoms, like sore throat and lymphadenopathy, and the serology was negative at the beginning of the disease. This lead to a more exhaustive investigation in order to exclude autoimmune and thyroid diseases and viral infections like hepatitis and HIV.

\section{P385 PRIMARY CAREGIVERS KNOWLEDGE ON HOME MANAGEMENT OF CHILDHOOD FEVER}

${ }^{1}$ Hemal De silva*, Shreenika De Silva ${ }^{2},{ }^{1}$ Kumudu Weerasekera. ${ }^{1}$ Ministry of Health, Colombo, Sri Lanka; ${ }^{2}$ University of Colombo, Colombo, Sri Lanka

\subsection{6/archdischild-2019-epa.731}

Introduction The objective was to assess the knowledge on childhood fever and knowledge and practices on home management of a febrile child among the primary caregivers; the socio-demographic predictors and the source of information associated with the knowledge and practices.

Methods A descriptive cross-sectional study was carried out in four randomly selected wards at the Lady Ridgeway Hospital Sri Lanka among 400 primary caregivers of children less than 12 years of age admitted with fever. A pre-tested, pre-coded, interviewer administered questionnaire was used.

Results Normal body temperature and temperature during fever were correctly mentioned by $46.2 \%$ and $35.8 \%$ of the study population respectively. Although $52.8 \%$ knew about a diurnal variation of normal body temperature, only $3.5 \%$ described it correctly. Fits were thought to be an effect of fever by $73.8 \%$. However, only $33.5 \%$ mentioned dehydration as an effect. Touching the skin is not different from using the thermometer for identification and monitoring of fever was a belief among $21.9 \%$ of the respondents. Mercury thermometer was identified as the best thermometer by $45.8 \%$, while it was $34 \%$ for digital. Paracetamol was not identified as the best antipyretic by $2.3 \%$. The helpfulness of blood tests before visiting a medical practitioner was mentioned by $48 \%$ of the respondents.

In practice, $60 \%$ of the study population used a thermometer for detection and/or monitoring of fever. The thermometer was used by $54.0 \%$ for identification of fever while $45.8 \%$ used the touch method. In monitoring of fever $59.5 \%$ used the thermometer while $40.5 \%$ used only the touch method.

Paracetamol was used by $99 \%$ of the study population to treat the febrile child at home. Non-Steroidal Anti-Inflammatory Drugs were also used by $1.5 \%$. The dose of paracetamol given to the child was too low for $6.5 \%$ while it was too high for $24.5 \%$. Awakening the sleeping child to administer antipyretics was practiced by $91.8 \%$. Dengue NS1 antigen test was done by $10.5 \%$ and a full blood count by $31.3 \%$ before seeking medical assistance. Statistically significant associations 